Zuerst ersch. in: Deutsche Zeitschrift für Philosophie, 44 (1996), 5, S. 759-775

\title{
Der Wert der Freiheit
}

\section{Von GOTTFRIED SEEBASS (Konstanz)}

\section{Freiheit - ein mißbrauchter Begriff}

Freiheit, wir wissen es, ist ein hohes Gut. Verbal sogar ein äuBerst hohes, gemessen jedenfalls an jenen vielzitierten, seit Aischylos oft varierten Erklärungen, wonach es besser sei zu sterben, als in Tyrannei oder Sklaverei zu leben, Wachsamkeit den „Preis der Freiheit" bilde oder nur der sich "Freiheit wie das Leben" verdiene, der sie sich täglich erobern muB. ${ }^{1}$ Wie weit die historische Wirklichkeit diesem Selbstbild wirklich entspricht. steht auf einem anderen Blatt. Doch noch im bloßen Lippenbekenntnis manifestieren sich geltende Werte. Nichts demonstriert dies eindringlicher als die ubiquitäre Verwendung des Wortes „Freiheit" zu Werbezwecken, ökonomischen wie politischen. Während die Pulverkaffee bereitende Schöne flötet "Ich bin so frei“, verspricht der rauchende Westmann uns den „Geschmack von Freiheit und Abenteuer". Während der Sozialist hoffnungsfroh seine „Brüder zur Sonne, zur Freiheit" ruft, verkündet sein konservativer oder liberaler Widerpart ungeniert, Freiheit und Sozialismus schlössen sich aus. In einem der Landtagswahlkämpfe des Jahres 1994 verwendete eine Partei sogar ein Plakat, auf dem nur noch die Wörter „Freiheit" und ,statt" gedruckt erschienen, während der nachfolgende politische Gegenbegriff handschriftlich eingesetzt war. ${ }^{2}$ Deutlicher kann man die Sinnentleerung eines Begriffes kaum dokumentieren, Die alltägliche, zumal politische Rede von „Freiheit* ist nahezu ebenso nichtssagend geworden wie die vom "Gluck", "Gemeinwohl" oder vom "Guten" überhaupt. All diese Worter sind inhaltsleere, positiv assozierte Chiffen, die gerade deshalb so gut zur Werbung geeignet sind, weil jeder das in sie eintragen kann, was er will.

Von der Sinnleere aber ist es nur noch ein Schritt bis zur rabulistischen Verkehrung ins Gegenteil. Schon Montesquieu hat das bemerkt und als Beispiel die Ansicht einiger Interessierter angeführt, „Freiheit" bestünde im Recht, Waffen zu tragen und Gewaltakte ungehindert begehen zu können. ${ }^{3}$ Goethe diagnostizierte sogar, rủckblickend offenbar auf die Epoche der Französischen Revolution und der Napoleonischen Kriege, daß3 man: „,niemals mehr von Freiheit reden hört, als wenn eine Partei die andere unterjochen will und

$1 \mathrm{Vgl}$. Aischylos, Agamemnon, V. 1364; J. W. Goethe. Faust II, V.1571 ff. Das geflügelte Wort vom "Preis der Freiheit" wird ohne Quellenangabe Thomas Jefferson zugeschrieben.

2 Vgl das einschlägige Foto im "Zeit-Magazin“", Nr. 38. 16.9. 1994.29.

3 Ch.-L.S. Montesquieu, De l'esprit des lois, Kap. XI,2. 
es auf weiter nichts angesehen ist, als daß Gewalt, Einfluß und Vermögen aus einer Hand in die andere gehen sollen." Und Aldous Huxley schließlich hat den Punkt auf die Spitze getrieben, wenn er einen seiner Romanhelden die sarkastische Feststellung treffen läßt, Menschen seien immer bereit, sich durch „magische" Wörter wie "Freiheit" selbst für die pseudotiefsinnige Paradoxie zu begeistern, daß das "eigentliche" oder ,wahre $X^{\text {* }}$ "Nicht-X" sei, ,wahre Freiheit" also z. B. Gefangenschaft."

Völig abwegig ist das nicht. Dennoch ist die von Huxley gegeiBelte radikale Begriffsverkehrung weniger kritisch, da sie dem reflektierten Betrachter offen entgegentritt. Wirkliche Demagogen sind raffinierter. Als die Wehrmacht 1941 in Rußland einfiel, lieB Goebbels der „Wochenschau“ eine Liedstrophe anhängen, die das Gezeigte deuten sollte und mit den Worten schloß: "Freiheit das Ziel, / Sieg das Panier! / Führer befiel, / wir folgen dir!" Welche Freiheit ist hier gemeint? Die Freiheit Rußlands etwa oder gar diejenige Deutschlands, die es durch einen Präventivschlag vor der Eroberung Stalins zu retten galt? Kaum. Denn niemand, der die Vorgänge seit 1938 verfolgt hatte und bei Verstand war, konnte verkennen, daß es hier nicht um Abwehr ging, sondern um eigene Machterweiterung. Zudem war die erklärte „Befreiung“ schwer mit der Forderung in Einklang zu bringen, sich dem ,Führerbefehl" bedingungslos zu unterwerfen. Nun wußte Goebbels natürlich, daß die meisten Kinobesucher solche Fragen nicht stellen wïrden. Aber er mußte sich auch bei den anderen darauf verlassen können, daß seine Rede von "Freiheit" nicht rabulistisch erschien und thre Deutungsfunktion damit einbüßte. Wie konnte er das?

Nun, es gibt auch hier einen relevanten, begrifflichen Hintergrund. Der Gedanke, daß wahre Freiheit autoritative Führerschaft nicht etwa aus-, sondern im Gegenteil einschließt, hat eine alte Tradition, auf die ich später noch einmal zurückkomme. ${ }^{6}$ Und selbst ihr Zusammenhang mit gewaltsamer Machtentfaltung ist keine NS-Erfindung. Montesquieus kritische Bemerkung über die Freiheit des Waffenträgers belegt das. Weit radikaler und politisch verhängnisvoller aber belegt es die positive Uberzeugung eines namhaften deutschen Philosophen, der "seinen Begriff von Freiheit " dahingehend bestimmt: „daß man die Distanz, die uns abtrennt, festhält. Daß man gegen Mühsal, Härte, Entbehrung, selbst gegen das Leben gleichgültiger wird. Daß man bereit ist, seiner Sache Menschen zu opfern, sich selber nicht abgerechnet. Freiheit bedeutet, daß die männlichen, die kriegs- und siegsfrohen Instinkte die Herschaft haben über andere Instinkte, zum Beispiel über die des ,Glücks*. Der freigewordene Mensch, um wieviel mehr der freigewordene Geist, tritt mit Füßen auf die verächtliche Art von Wohlbefinden, von dem Krämer, Christen, Kühe, Weiber, Engländer und andere Demokraten träumen. Der freie Mensch ist Krieger. ${ }^{*}$ ?

4 J. W. Goethe, West-östlieher Divan. Noten und Abhandlungen, Nachtrag.

5 A. Huxlcy, Eyeless in Gaza, London 1936, 122-124, - Den Hinweis auf diese, in der Literatur mehrlach zitierte Passage verdanke auch ich C.L. Stevenson, Ethics and Language, New Haven $1944.214 \mathrm{f}$.

6 Das gilt für die theologische Tradition in Europa ebenso wic für die ständische germanische Rechtsordnung. Für die letztere vgl. Ch. Dipper, Artikel „Freihcit IV;: Ständische Freiheit: Jura ct libertates", in: D. Brunner u. a. (Hg.), Geschichtliche Grundbegriffe, Bd. II, Stuttgart 1975, 447 ff. Für die theologische Tradition vgl. Anm. 34.

7 F. Nietzsche, Götzen-Dämmerung, IX. 38. 
An eine solche Definition von „Freiheit" konnte Goebbels problemlos anknüpfen. Gewiß, auch innerhalb des nicht eben durch seine Besonnenheit ausgezeichneten Euvres von Nietzsche markiert sie ein Extrem, das man dem Autor und seinen Bewunderern nicht uneingeschränkt zurechnen sollte. Sie verdeutlicht jedoch auf besonders drastische Weise die begrifflichen Schwierigkeiten, vor denen wir stehen. Die Usurpation des sinnentleerten Positiv-Wortes "Freiheit" durch fremde Inhalte beginnt eben nicht erst mit der politischen Propaganda. Ihre geistigen Wurzeln liegen tiefer. Und das betrifft auch Traditionen, die uns seriös entgegentreten und deren Einfluß wir uns, wenn wir ihn überhaupt registrieren, nicht ohne weiteres entziehen können. Das gilt für die Inhalte und für die Wertungen natürlich erst recht. Die scheinbar selbstverständliche Rede vom Wert der Freiheit bedarf deshalb, mehr noch als andere vermeintliche Selbstverständlichkeiten, der tieferdringenden, kritischen Reflexion. Insofern ist sie ein Thema der Philosophie, zu dem ich mit diesem Aufsatz einen Beitrag zu leisten versuche.

\section{Der deskriptive Gattungsbegriff}

Wie können wir dabei vorgehen? Die wichtigste Aufgabe ist eine Präzisierung des Freiheitsbegriffs. Denn nur wenn man Klarheit darüber hat, was Freiheit ist, kann man sinnvoll danach fragen, ob sie wertvoll ist. Dazu muß die deskriptive Komponente der Rede von "Freiheit" strikt von der evaluativen getrennt werden. Vergessen wir also für einen Augenblick, daß das Wort wertbesetzt ist, und konzentrieren uns ganz auf seinen Gehalt. Auch dieser kann offenbar sehr verschieden ausfallen. Der deskriptive Freiheitsbegriff muß deshalb nicht nur präzisiert, sondern auch intern differenzient werden. Einige Verwendungsweisen werden sich danach als willkürlich oder rabulistisch ausgrenzen lassen, andere sich als Grenzfälle oder begriffliche Derivate erweisen. Um sie jedoch als solche erkennen zu können, müssen wir wissen, wo das begriffliche Zentrum liegt. Fragen wir deshalb zunächst, ob es so etwas wie eine Grundbedeutung, einen Gattungsbegriff der "Freiheit" gibt, von dem alle spezielleren Begriffe abhängen.

Manche Autoren bestreiten das. So z. B. der Ökonom Friedrich von Hayek, einer der Hauptvertreter des neueren Wirtschaftsliberalismus. „Krudester philosophischer Realismus" sei es, Gleichheit des Begriffs aus der Gleichheit der Wörter abzuleiten, während diese in Wahrheit eine „verbale Falle" sei, eine "Quelle gefährlichen Unsinns", die die „absurdesten Konklusionen" heraufbeschwört. ${ }^{8}$ Die Vehemenz dieser Attacke ist auffällig. Sie ist kaum dazu angetan, falsche Begrifflichkeiten zurechtzurücken, sondern weckt ihrerseits den Verdacht interessierter Begriffsverdrehung. Ich werde das später substantiieren. Zunächst geht es mir nur um die Feststellung, daß Hayek sich mit ihr weit von der philosophischen Tradition entfernt. Sein Angriff nämlich richtet sich, näher betrachtet, weniger gegen die Suche nach einem Gattungsbegriff der „Freiheit" uberhaupt als gegen einen bestimmten, traditionellen Vorschlag, sc. das Konzept der Handlungs- bzw. Hindernisfreiheit. ${ }^{7}$ Handlungsfreiheit besagt, kurz gefaßt, daß man handeln kann, wie man will.

8 F. A. Hayek, The constitution of liberty, London 1960, 18 f.; Übersetzung vom Verfasser.

9 Hayek (ebd., 17, Anm. 18-19) füht als Vertreter der Handlungsfreiheit lediglich Russell an, als Vertreter der Hindernisfreiheit Hobbes und (in Anm. 24) zwei neuere Autoren. Ob Hayek sich seines Gegensatzes zur Hauptströmung der neuzeitlichen Philosophie bewuBt war, geht aus dem 
Dieser Freiheitsbegriff, der sich wenigstens bis zu Augustin und in weniger prägnanter Form bis ins klassische Griechentum zurückverfolgen läßt, wird von den meisten Philosophen vertreten. Viele haben ihn sogar als den einzig sinnvollen aufgefaßt und zum Gattungsbegriff erhoben. Das ist tatsächlich zweifelhaft. Nicht jedoch aus dem von Hayek angegebenen Grund, daß er in sich falsch konzipiert oder die ganze Idee einer begrifflichen Integration der Rede von „Freiheit" verfehlt wäre. Der Grund ist vielmehr, daß der als solcher völlig korrekte Begriff der Handlungsfreiheit zwar allgemein, aber nicht allgemein genug ist, um als Gattungsbegriff fungieren zu können. Diese Funktion erfüllt erst der Begriff der Hindernisfreiheit, der den der Handlungsfreiheit als seinen wichtigsten Sonderfall mitumfaßt.

Hobbes und Schopenhauer vor allem waren es, die dies erkannt und den Kern unserer Rede von "Freiheit" richtig bestimmt haben. ${ }^{10}$ Beide haben dabei zu Recht darauf abgestellt, daß diese Rede sich nicht nur auf Menschen bezieht, die wollen und willentlich handeln können. Sie betrifft auch andere Lebewesen und sogar leblose Dinge. So reden wir etwa vom „freien Fall" oder „freien Lauf eines Flusses", von „freien Valenzen ", ,bleifreiem Benzin" oder Tieren in „freier Wildbahn". Worauf aber grundet sich diese Rede? Offenbar darauf, daß in all diesen Fällen das, was hier ,frei" genannt wird, frei ist von charakteristischen Hindernissen. Frei ist der Lauf eines Stroms, wenn er weder durch natürliche noch durch künstliche Hindernisse in ein bestimmtes Flußbett gezwängt wird. Tiere in freier Wildbahn sind frei, weil sie sich, ungehindert durch menschliche Domestizierung oder Umzäunung, frei bewegen und fortpflanzen können. Und so weiter. Entsprechendes gilt für das freie Handeln von Menschen. Der Gefangene oder Gefesselte ist unfrei, weil er gehindert wird, sich zu bewegen, wie er will. Mehr noch. Auch die Freiheit des handlungsbestimmenden Wollens und Wünschens, also der Kern der Willensfreiheit, die der Begriff der Handlungsfreiheit nicht zu erfassen vermag, wird als Hindernisfreiheit verständlich. So ist der durch Waffengewalt oder sonstige Drohungen anderer Menschen zur Willfährigkeit Gezwungene unfrei, weil er zwar nicht am willensgemäßen Handeln, wohl aber in seiner vorbereitenden Willensbildung signifikant gehindert wird. Ähnliches gilt für Notlagen ohne Fremdeinwirkung, sowie in besonders radikaler Form auch für jene Willensunfreiheit, die durch Drogensucht oder psychische Abhängigkeit entsteht.

Naturlich müßte der Anspruch, daß jede sinnvolle Rede von „Freiheit" letztlich auf den Begriff der Hindernisfreiheit zurückführt, eingehender geprüft und gerechtfertigt werden. Ich muß mich mit diesen wenigen Beispielen begnügen und den Leser bitten, mir das Konzept der Hindernisfreiheit, über das ich an anderer Stelle mehr gesagt habe ${ }^{11}$, ohne weitere Argumente zu schenken. Auch seine weitere Präzisierung und Differenzierung kann ich hier nicht in Angriff nehmen. Ein Präzisierungsschritt allerdings ist für unsere Frage unumgänglich. Wir müssen Klarheit darüber haben, daß sich die Rede von einer „Hinderung“

Text nicht eindeutig hervor. Wenn ja, ist seine skizzenhafte Behandlung als ein Versuch zu werten, ihre Bedeutung herunterzuspielen.

10 Th. Hobbes, De cive, Kap. 9; Leviathan, Kap. 21; Of liberty and necessity, in: Works, ed. Molesworth, vol. IV, 273.275f; A. Schopenhauer, Preisschrift über die Freiheit des Willens, Abschn. I.

11 G. SeebaB, Wollen, Frankfurt/M. 1993, Kap. VI; Freiheit und Determinismus, in: Zeitschrift für philosophische Forschung 47 (1993), 1-22, 223-245; Die konditionale Analyse des praktischen Könnens, in: Grazer philosophische Studien 49(1995), 1-28. 
immer auf zwei zentrale Kriterien gründet. Erstens impliziert sie die Existenz eines Möglichkeitsspielratms, der dem Ungehinderten uneingeschränkt offensteht und dem Gehinderten nicht. Hier liegt der primäre Ansatzpunkt für die Graduierung der Freiheit. Je größer der umfassende Spielraum ausfällt sowie die Anzahl der Möglichkeiten, die dem Betreffenden in ihm zugänglich sind, desto größer ist, prima facie, auch seine Freiheit.

Das aber ist noch nicht alles. Die bloße Tatsache eines verringerten Möglichkeitsspielraums nämlich bedeutet nicht unbedingt eine Hinderung. Hinzukommen muß als zweites Kriterium, daß die verschlossenen Alternativen dem Gehinderten eigentlich zugänglich sein sollten. Freilich: was heißt das? Statt von der "Eigentlichkeit" ihres Offenstehens könnten wir auch davon sprechen, daß die Offenheit dem. der gehindert wird, ,wesensgemä $\beta^{*}$ wäre oder daß ihre Verschlossenheit "wider seine Natur" ist. Doch es ist klar, daß diese Auskunft noch nicht befriedigt. Denn was ist ,natur" oder "wesensgemäB"? Ich werde hier nicht den Versuch machen, diese notorisch schwierige Frage generell zu beantworten. Ich beschränke mich vieImehr darauf, die Signifikanz des Kriteriums der "Natürlichkeit“ oder :Wesensgemäßheit" beispielhaft zu erläutern.

Erstens: Daß ich nicht so schnell schwimmen kann wie Franziska van Almsick und nicht so gut Geige spiele wie Arthur Grumiaux, begrenzt meinen Handlungsspielraum. Kann ich doch weder Medaillen sammeln noch den Grand Prix du Disque bekommen. Gleichwohl bin ich als so Eingeschränkter nicht unfrei. Daß ich nur eine lahme Ente im Wasser bin, stört mich überhaupt nicht. Eher schon stören mich meine violinistischen Defizite. Aber auch sie sind, wenngleich bedauerlich, kein entscheidendes Freiheitshindernis für einen musikalischen Amateur, der seinen Abstand zu den wirklichen Könnern erkannt und ein für allemal anerkannt hat.

Zweitens: Sind Männer unfrei, weil sie Männer sind und keine Frauen? Transsexuelle empfinden es so. Und gewisse Äußerungen von feministischer Seite legen die Auffassung nahe, daß die männliche Lebensauffassung per se am Leben vorbeigeht. Daran mag etwas Richtiges sein, zumindest wenn man die ,männlichen Instinkte" Nietzsches im Auge hat. Doch die Mehrheit der Männer sieht das offenbar anders. Sie fühlen sich durch ihre Männlichkeit nicht in ihrer Freiheit eingeschränkt. Umgekehrt erleben viele Frauen ihr Frausein als freiheitsbeschränkend, und daran ist zweifellos vieles richtig. Aber auch hier geht es eigentlich nicht um die Geschlechtszugehörigkeit selbst, sondern um die Beschränkungen, die in der Gesellschaft mit ihr verbunden sind und die in anderer Form auch für Männer bestehen können. Diese Beschränkungen werden als nicht wesensgemäße soziale Kontingenzen aufgefaBt, die den Freiheitsspielraum beschneiden. Das Faktum der Geschlechtlichkeit selbst dagegen, einschließlich ihrer Grenzen, wird normalerweise als naturlich und wesensgemäß anerkannt.

Und noch ein drittes, extremes Beispiel: Als rationale Wesen haben wir offenbar nicht die Möglichkeit, zu glauben oder sinnvoll zu behaupten, daB ein und derselbe Sachverhalt gleichzeitig wahr und falsch ist. Sind wir deshalb, anders vielleicht als der Gott Wilhelm von Ockhams ${ }^{12}$, in unserer Freiheit eingeschränkt? Sicher nicht, auch wenn manche mit dem Gedanken gespielt haben, den Satz vom Widerspruch außer Kraft zu setzen. Elementare logische und grammatische Regeln enthalten Beschränkungen, die nicht als Hinde-

12 Vgl. W. v. Ockham, Tractatus de praedestinatione et de praescientia Dei et de futuris contingentibus. Ass. 7. 
rung zu verstehen sind, sondern im Gegenteil als Voraussetzung für den Bezug auf relevante Möglichkeitsspielräume. Ähnliches gilt für andere Formen mutmaßlichen „rationalen Zwangs", wenn auch gewiB nicht für alle. Denn natürlich müssen nicht alle Regeln, die eine Gesellschaft der , wissenschaftlichen “ oder „, kulturellen Vernunft" zurechnet, absolut gültig sein. Einzelne Menschen, namentlich Künstler und kreative Wissenschaftler, haben sie deshalb immer wieder in Frage gestellt und versuchsweise, mit oder ohne Erfolg, aufgehoben.

Wo die Grenzen solcher Erweiterungen liegen, muß hier ebenso offenbleiben wie das gesamte Problem der Bedingungen, unter denen das abstrakte Kriterium der ,Natürlichkeit" oder „Wesensgemäßheit" im Einzelfall konkretisiert wird. Wichtig ist jedenfalls, daß es ins Spiel kommt. Die Rede von einem „Gehindertsein“ und somit von „Freiheit" hängt nicht nur von der GröBe des Spielraums ab, sondern auch davon, ob die verschlossenen Möglichkeiten das, was unfrei und gehindert sein kann, in seiner (wie ich im folgenden summarisch sagen möchte) ,wesensgemäß̉en Entaltung " beeinträchtigen. Die Konkretisierung dieses Abstraktums geht Hand in Hand mit der Spezifizierung der Freiheitsbegriffe. Speziellere Begriffe unterscheiden sich durch die Art und die Anzahloffener Möglichkeiten, durch die Gründe für ihre Offenheit oder Verschlossenheit und durch die Arten von Individuen, die sie betreffen. Die Freiheit eines ,frei fallenden“ Steins ist, trotz des gemeinsamen begrifflichen Rahmens, natürlich eine wesentlich andere als die Freiheit eines Menschen, der sich in seinem Denken, Wollen und Handeln ungehindert entfalten kann. Was bei leblosen Objekten als ,wesensgemä $B^{* *}$ oder ,natürlich" gilt, und ob sie überhaupt so beschrieben werden, ist theorieabhängig, also letztlich bedingt durch Menschen, die sich von außen auf sie beziehen. Beim Menschen selbst ist das wesentlich anders. Hier wird die Frage, was für ihn ,wesensgemä $B^{*}$ ist, nicht oder doch nicht in letzter Instanz von außen entschieden, sondern von den Betreffenden selbst, auch wenn Krankheit und Unmündigkeit diese Entscheidungskompetenz temporăr aussetzen können. ${ }^{13}$ Weitere gravierende Differenzen kommen hinzu. Ich muB es bei diesem (zwangsläufig skizzenhaften) Blick auf die deskriptive Komponente der Rede von „Freiheit" belassen und wende mich nun der evaluativen zu. also der für diesen Aufsatz zentralen Frage des Werts der Freiheit.

\section{Extrinsische und intrinsische Werthaftigkeit}

Beginnen wir mit der Rede vom "Wert". Welchen Sinn hat sie in unserem Zusammenhang? Zwei Deutungen sind in Betracht zu ziehen. Einmal kann ein extrinsischer Wert gemeint sein, also ein Wert, den etwas dadurch erhält, daß es ein Mittel zu etwas anderem darstellt, das wertvoll ist. Auch eine weggeworfene, verrostete Büchse kann wertvoll

13 Das gilt ubrigens auch mit Bezug auf jene Notwendigkeit einer "Erziehung zur Freiheit“, für die F. Tallár kürzlich in der "Deutschen Zeitschrift für Philosophie" eine Lanze gebrochen hat (Bd. 44. 1996, Heft 2, $229 \mathrm{ff}$.). ..Mut zur Erzichung" darf nicht muwillige Vergewaltigung heiBen. Legitimiert ist solcher Mut nur im Vorgriff auf die zu erwartende Zustimmung des zu erziehenden Menschen. Dessen Entscheidungskompetenz wird durch die temporäre. stellvertretende Tätigkeit des Erziehers nicht aufgehoben. Auch Tallár scheint das letztlich nicht anders zu sehen, wenngleich er diesen für mich entscheidenden Punkt nur halbherzig einräumt, versteckt etwa in ein Fichte-Zitat (ebd., 236) und in beiläufige Erklärungen uber die Möglichkeit des Verfehlens der „wirklichen Interessen“ anderer (ebd.. 233). 
werden, wenn sie als zweckentfremdetes Ersatzteil bei einer Notreparatur unentbehrlich ist. Extrinsische Werte aber setzen Werte voraus, die sich letztlich nicht mehr von anderen ableiten lassen. Am Ende der Kette stehen intrinsische Werte, die ihren Trägern als solchen zukommen. Intrinsische Werte müssen nicht absolut wertvoll sein. Sie können auch gleichrangig nebeneinander stehen oder sich anderen Werten unterordnen, ohne vollständig von ihnen abhängig zu sein. Ein delikates Essen verliert seinen eigenen Wert nicht dadurch, daß es gegebenenfalls dem höheren Gut der Gesundheit weichen muß. Und die Gesundheit bleibt ein Gut. auch wenn wir sie manchmal für andere Güter aufs Spiel setzen. Welcher der beiden Wertbegriffe also. der intrinsische oder der extrinsische. ist für die Rede vom ,Wert der Freiheit" konstitutiv?

Kann sie als rein extrinsischer Wert verstanden werden, ohne intrinsische Wertkomponente? Teilweise wird das vertreten. Vor allem "liberale" Politiker und Vertreter der "freien" ( = maximal deregulierten) Marktwirtschaft versuchen, die Forderung nach weitgehender staatlicher Nichtintervention damit zu rechtfertigen, daß maximale soziale Freiheit, alles in allem genommen, das beste Mittel zur Wohlfahrt sei. Das impliziert die Bereitschaft, sie u. U. fallen zu lassen. Der extrinsische Wert der Freiheit muß, wenn bessere Mittel verfügbar sind, dem intrinsischen Wert der Wohlfahrt weichen. Das kann im Extremfall heißen, daß der Staat, wenn dies am effektivsten ist, seine Bürger auf genetischem oder direktem neurostimulativen Wege zu wohlfahrtsoptimalem Verhalten bringt. Das ist bizarr. aber vom Ansatz her konsequent. Es liegt deshalb eine gewisse Ironie darin, Positionen wie die gerade erwähnten, die Freiheit nicht einmal als intrinsischen Wert auffassen, geschweige denn als hochrangigen oder absoluten, als "Liberalismus" anzusprechen. In Wahrheit stellen sie Spielarten des Utilitarismus dar, solche nämlich, die sich über das zentrale Prinzip der Nutzenmaximierung hinaus auf ein bestimmtes Mittel zu seiner Erfullung festgelegt haben.

Letzteres aber ist eine rein empirische Frage, die rein wissenschaftlich zu entscheiden ist, sine ira et studio. Das steht erneut im ironischen Widerspruch zu der Leidenschaft des politischen Streits um den extrinsisch begründeten sogenannten „Liberalismus". Eine historische Eselei, die leicht auszuräumen sein sollte? Nein, sondern die naheliegende Folge weithin bestehender Unklarheit und partiell bewuBter Verunklarung. Ein kurzer Blick auf die Hintergründe verdeutlicht das. Zunächst sind die empirischen Evidenzen, trotz alles wirtschaftswissenschaftlichen Fleißes und eines ausgesetzten Nobelpreises, bislang alles andere als eindeutig und sicher. Gewiß, daß eine Planwirtschaft nach sowjetischem Vorbild ineffektiv ist, wird heute niemand in Abrede stellen. Aber die Frage, welche Form ökonomischer Deregulierung sinnvoll ist, bietet immer noch breiten Raum für ideologische Glaubenskriege. Diese entstehen nicht nur aus Mangel an Wissen. Sie entstehen auch aus der Unklarheit über den deskriptiven Freiheitsbegriff und der mangelnden Rücksicht auf verschiedene Arten der Freiheit. Vor allem aber gründen sie in der notorischen Vagheit des zugrundegelegten intrinsischen Werts, sc. "Wohlfahrt" oder "Nutzen". Denn worin bestehen diese? In einem formalen Abstraktum etwa wie unserem Bruttosozialprodukt, losgelöst von allen Fragen seiner gerechten Verteilung und seiner realen, materialen Gegenwerte. kulturelle und ökologische eingeschlossen? Auch der eingefleischteste Marktwirtschaftler wird das nicht zugeben wollen. Stellt man sich diesen Fragen jedoch. wird sogleich klar, daß uber sie weder Einigkeit noch konzeptionelle Klarheit herrscht. Ohne einen bestimmten Begriff des ,Wohls" jedoch, das als intrinsisch wertvoll gilt, und ohne 
gesicherte empirische Grundlagen sind alle Aussagen über den abgeleiteten Wert der Freiheit wertlos. Da ich diese Vorraussetzungen bis auf weiteres nicht gegeben sehe, werde ich das extrinsische Verständnis der Wertfrage beiseite setzen.

\section{Unhintergehbarkeit und externe Relativierbarkeit}

Der klassische Liberalismus, der die westlichen Demokratien geprägt hat und im 19. Jahrhundert auch auf dem Kontinent die politisch treibende Kraft war, war hier auch anderer Meinung. Er hat die Freiheit nicht nur als extrinsischen Wert verstanden, sondern als intrinsischen, teilweise sogar absoluten. Diese Auffassung erscheint plausibel, wenn man sich klar darüber ist, was die Rede von „Freiheit" im Kern besagt. Denn daß die wesensgemäBe Entfaltung von Menschen nur deshalb wertvoll sein sollte, weil sie ein Mittel zur Wohlfahrt ist, erscheint mehr als seltsam. Eher gilt hier das Umgekehrte. Prüfen wir deshalb die These vom Wert der Freiheit in ihrer intrinsischen Deutung.

Können wir sagen, daß Freiheit ein absoluter Wert ist? Kant hat das offenbar angenommen, wenn er in der Einleitung zur „Rechtslehre" die Freiheit als das ,einzige, ursprüngliche, jedem Menschen kraft seiner Menschheit zustehende Recht" hinstellt. ${ }^{14}$ Doch das skizzierte Konzept der Hindernisfreiheit läßt uns sogleich erkennen, daß eine so pauschale Antwort auf die Wertfrage unmöglich ist. Die unspezifizierte Rede von einem Grundrecht oder absoluten Wert "der Freiheit" macht wenig Sinn, da sie nicht zu erkennen gibt. wer hier woran und aus welchen Gründen gehindert sein kann. Setzt man jedoch spezielle Freiheitsbegriffe ein, zeigt sich, daß diese keineswegs absolute Wertgeltung haben, sondern relativierbar sind. Auch Kant spricht, näher betrachtet, nicht von einem abstrakten Freiheitsrecht, sondern von einem angeborenen Recht auf Unabhängigkeit von der ,nöthigenden Willkür" anderer, das zudem durch die Bedingung eingeschränkt ist. daß sie, wie er formuliert, "mit jedes Anderen Freiheit nach einem allgemeinen Gesetz zusammen bestehen kann". 15 Und noch deutlicher wird ihre Relativierbarkeit in den Formulierungen moderner Verfassungen und Menschenrechtserklärungen. Denn hier wird gewöhnlich explizit statuiert, nicht nur daß, sondern auch wie und zugunsten welcher anderen Werte das Grundrecht der Freiheit eingeschränkt werden kann. ${ }^{16}$

Ob die dabei genannten anderen Werte dem Wert der Freiheit tatsächlich gleichrangig oder ubergeordnet sind, kann man mit Grund bezweifeln. Ich werde darauf zurückkommen. Aber auch unabhängig davon läßt sich die Relativierbarkeit spezieller Freiheitsbegriffe leicht unter Beweis stellen. Denn zahllose konkrete Freiheiten relativieren sich wechselseitig. Daß man die grenzenlose, doch ungesicherte Freiheit des „Naturzustands“ aufgeben muß, um gesicherte Freiheiten allererst zu bekommen, ist der Grundgedanke des neuzeitlichen Kontraktualismus. Potentielle Gewalttäter müssen an dieser Form ihrer "Selbstentfaltung" gehindert werden, damit die von ihnen Bedrohten sich ungehindert entfalten können. Gäbe es tatsächlich „freie Fahrt für freie Bürger", wie sie der Slogan dümmlich beruft, wiirde das resultierende Verkehrschaos unsere Bewegungsfreiheit als-

14 I. Kant. Metaphysik der Sitten, Einleitung in die Rechtslehre, AA V1, 237.

15 Ebd.

16 Einschlägige Texte sind abgedruckt in W. Heidelmeyer ( $\mathrm{Hg}$.), Die Menschenrechte, Paderborn ${ }^{3} 1982$, vgl, insbesondere $94,276,280 \mathrm{ff}, 297,305$ f, 310. 
bald beseitigen. Insofern ist die von Unbedachten vielleicht als reaktionär oder rabulistisch empfundene Erklärung von Locke, daß es Freiheit nur in gesetzlicher Bindung gebe, und daß der Satz, daß keine Regierung absolute Freiheit erlaubt, analytisch wahr sei ${ }^{17}$, auf konkrete Freiheiten in der Gesellschaft bezogen lediglich eine Binsenweisheit.

Natürlich bedeutet das nicht, daß alle Freiheiten in gleicher Weise relativierbar sind. Es gibt gravierende Unterschiede. Charles Taylor etwa hat eine Rangfolge hergestellt $z$ wischen freiheitsbeschränkenden Verkehrsregeln, Freizügigkeitsbeschränkungen und Meinungsunfreibeit in moralischen und religiösen Dingen. ${ }^{18}$ Ebenso hat das deutsche Bundesverfassungsgericht in der Ausgestaltung von Artikel $2 \mathrm{GG}$ eine dreifache Unterscheidung getroffen zwischen der ,innersten Sphäre" menschlicher Freiheit, einer ,weiteren Privatsphäre" und einer noch weiter gefaßten ,Sozialsphäre". 19 Solche Einteilungen sind relativ grob. Sie sind nur Ansätze zu einer differenzierteren, internen Abwägung. Offen bleibt zudem. ob nicht bestimmte Arten der Freiheit als absolute, prinzipiell nicht relativierbare Werte zu verstehen sind. Denkbar wäre das wohl am ehesten bei jener „Freiheit der Gedanken", die nach der Volksweisheit niemals verlorengeht, im Zeitalter psychologischer "Gehirnwäsche" und neuraler Manipulationsmöglichkeiten aber durchaus gefährdet erscheint.

Ich möchte diesen direkten, stärkeren Sinn der Rede vom, absoluten Wert" hier jedoch nicht aufgreifen. Ich konzentriere mich auf einen schwächeren Sinn, wonach Freiheit deshalb, absolut wertvoll" ist, weil sie, wenn überhaupt, nur zugunsten anderer Freiheiten eingeschränkt werden kann, nicht durch sonstige Werte. Diese Auffassung möchte ich, auf den Menschen beschränkt, als "Unhintergehbarkeitsthese" bezeichnen. Sie ist nicht unplausibel und in der Literatur öfter vertreten worden, z. B. von John Rawls. ${ }^{20}$ lch möchte die Frage des Werts der Freiheit deshalb so weiterverfolgen, daß ich speziell danach frage, ob die Unhintergehbarkeitsthese haltbar ist, $\mathbf{d}$. h. ob es Konkurrenzwerte zur Freiheit gibt, die sie extern relativieren. Dabei geht es nicht nur um Werte, die explizit als Konkurrenten geltend gemacht werden. Kritischer noch sind Fälle, in denen es scheinbar um die Relativierung einer durch eine andere Freiheit geht, tatsächlich jedoch um eine Relativierung von außen, die dadurch verschleiert wird, daß der eine Freiheitsbegriff, wie wir zu Anfang gesehen hatten, verdreht oder durch fremde Merkmale modifiziert wird. Beide Formen der Konkurrenz möchte ich kurz beleuchten.

\section{Gleichheit und andere explizite Konkurrenzwerte}

Werfen wir zuerst einen Blick auf explizite Konkurrenzwerte. Sinnvoll ist es hier, mit dem neben der Freiheit zweiten zentralen Schlagwort der Französischen Revolution zu beginnen, der Gleichheit. ${ }^{21}$ Sie bildet, wie die Freiheit, ein Kernstück moderner Verfassungen

$17 \mathrm{Vgl}$. J. Locke, Second treatise on government, $\$ 22.57$ : An Essay concerning human understanding, IV, 3. 18 .

18 Ch. Taylor, Der Irrtum der negativen Freiheit, in: (ders.), Negative Freiheit?. Frankfurt/M. 1988, $128 \mathrm{f}$.

$19 \mathrm{Vgl}$. dazu R. Alexy, Theorie der Grundrechte, Frankfurt/M. 1986, $326 \mathrm{ff}$.

20 J. Rawls, A theory of justice, Oxford 197I, ch. IV.

21 Die Briderfichkeil ist ihrem Gehalt nach ebenso wie in ihrem relativen Gewicht umstritten. Sie wurde zwar in Artikel 1 der UNO-Menschenrechtserklärung von 1948 unbestimmt aufgegriffen, 
und Grundrechtskataloge und scheint prima facie gleichrangig neben ihr zu stehen. In der politischen Auseinandersetzung zwischen "Liberalisten" und "Sozialisten" stellt sich das oft sogar so dar, als seien Freiheit und Gleichheit originäre Gegensätze und ließen sich nur auf Kosten der jeweils anderen realisieren. Doch das ist ein prinzipielles, begriffliches Mißverständnis.

Richtig ist, daß Gleichheit nicht auf Freiheit zu reduzieren ist, sondern ein eigenständiges Merkmal bildet. Beide Begriffe aber liegen nicht auf derselben Ebene und lassen sich nicht gegeneinander aufrechnen. Denn anders als das der Freiheit betrifft das Merkmal der Gleichheit nicht die Frage, was wertvoll ist, sondern die Frage, wer alles in den GenuB gegebener Werte kommt. Angewandt auf die Freiheit in der Gesellschaft besagt die Gleichheitsforderung einfach, daß alle Mitglieder gleichermaßen in ihren Genuß kommen sollen. Dies ist der Hintergrund für die klassische Formel, wonach die Freiheit des einzelnen ihre Grenzen an der der anderen findet. Man kann politisch darüber streiten, ob die Gleichheitsforderung sich auf alle Arten von Freiheit und alle Individuen, in oder außerhalb einer Gruppe, erstrecken sollte, oder nur auf bestimmte.22 Aber es ist absurd zu sagen, Freiheit und Gleichheit seien Konkurrenzwerte, die gegeneinander abzuwägen sind. Kant hat das richtig gesehen und seine These von der Freiheit als einzigem Grundrecht deshalb mit Selbstverständlichkeit, ohne Hinweis auf einen etwaigen Wertekonflikt, an die Gleichheitsforderung seines kategorischen Imperativs gebunden. ${ }^{2.3}$ Gleichheit also, so weitgespannt man sie fassen und so hoch man ihren Wert auch immer veranschlagen mag, kann die Unhintergehbarkeitsthese nicht erschüttern.

Doch welche anderen, explizit konkurrierenden Werte könnten es? Isaiah Berlin hat auBer der Gleichheit, die wir jetzt ausscheiden können, Gerechtigkeit, Glück, Sicherheit und öfentliche Ordnung als Konkurrenzwerte angeführt ${ }^{24}$ Moderne Verfassungen und Menschenrechtserklärungen nennen Sicherheit, Gesundheit, Allgemeinwohl und Nicht-

taucht in den zentralen historischen Dokumenten aber nicht auf und bietet daher auch von der Ouellenlage her kein besonders schlagendes Beispiel für einen Konkurrenzwert.

22 Dieser Streit ist sehr real und läßt sich nicht mit Hilfe eines veränderten Freiheitskonzepts weginterpretieren. R. Forst dagegen hat in einem Beitrag in der. .Deutschen Zeitschrift für Philosophie". Bd. 44, Heft 2, $211 \mathrm{ff}$., geltend gemacht, daß das Problem der sozialen Gleichverteilung des nach seiner Meinung inexistenten "Gutes der Freiheit" sich gar nicht mohr stellen würde. wenn man der Tatsache Rechnung trüge, daß Freiheiten „allsin durch die wechselseitige und legitime Anerkennung zwischen Personen und die Zuerkennung von Rechten" entstehen (ebd., 213 und passim).

Ich glaube nicht, daß diese letztere Behauptung richtig ist. Doch selbst wenn sie zuträfe, würde sie allenfalls die Genese soztaler Freiheiten verständlich machen, über sie setbst aber nichts Entscheidendes aussagen. Daß̉ es sich nicht um verteilbare Güter handelt, bliebe ebenso offen wie die Fragen, wem welche Freiheiten zugewiesen werden und warum diese Zuweisung wechselseitig erfolgen soll. Forsts gutgemeinter, sympathischer Versuch, einen reicheren Begriff der politischen Freiheit ,.jenseits der Opposition zwischen negativen und positiven, liberalen, republikanischen oder sozial-egalitären Freiheitskonzeptionen" einzuführen (ebd., 226), muß deshalb unbefriedigend und für seine Gegner unüberzeugend bieiben - solange zumindest, als er sich nicht auf die systematische, differenzierte Beantwortung der desk riptiven begrifflichen Fragen und Wertfragen einläßt, von denen jeder Versuch dieser Art abhängt.

23 Kant, Metaphysik der Sitten. a. a. $O$.

24 I. Berlin. Two concepts of liberty, in: ders., Four essays on liberty. Oxford 1969. $52 \mathrm{ff}$. 
verletzung geltender Moralvorstellungen. ${ }^{25}$ Doch es ist mehr als zweifelhaft, daB die Unhintergehbarkeitsthese dadurch gefährdet wird. Gerechtigkeit ist ein Wert, der ähnlich wie Gleichheit quer zur Freiheit liegt und nicht gegen sie angefuhrt werden kann. Sicherheit und offentliche Ordnung wiederum sind Bedingungen sozialer Freiheit. Die politische Philosophie von Hobbes bis Popper hat das so oft und unmißverständlich herausgestellt, daß der politische Streit um diesen vermeintlichen Gegensatz seltsam anmutet. Was hier tatsächlich gegeneinander steht, ist der Gewinn an Freiheit, den sicherheitsfördernde staatliche Maßnahmen mit sich bringen. und der gleichzeitige Freiheitsverlust an anderer Stelle. Der Skylla der Freiheitsvernichtung durch die Allgegenwart des organisierten Verbrechens oder unkalkulierbare nukleare oder gentechnische Risiken steht die Charybdis des omnipräsenten Polizei- und Schnüffelstaats gegenüber. Beidemal aber ist es die Freiheit der Bürger, die es zu schützen gilt. Ebenso läßt sich der Schutz der Gesundheit als eine Form des Freiheitsschutzes begreifen, gegeben, daß Menschen sich, privat wie öfentlich, nur als gesunde ,wesensgemäß entfalten" können (vgl. S. 764).

Echte Konkurrenzwerte zur Freiheit könnten sich allenfalls hinter den Chiffren des Glïcks, des Allgemeinwohls und der geltenden Moral verbergen. Doch solange diese nicht spezifiziert und als eigenständige Werte gerechtfertigt werden, bleibt die Sache dubios. Heinrich von Treitschke z. B., der in jüngeren Jahren tatsächlich so etwas wie ein Liberaler war, hat darin über Liberalisten wie Humboldt und Mill hinausgehen wollen, daß er die Herbeiführung eines (wie er sich ausdrückt) "schönen und guten Gesamtdaseins" als gleichberechtigte staatliche Aufgabe neben der Sicherung persönlicher Freiheit betrachtet. ${ }^{26}$ Das klingt vertraut, und zwar nicht nur im Blick auf historische Kollektivisten wie Rousseau. Hegel und Marx. Der bei Treitschke schon terminologisch offenkundige Rückgriff auf antike Vorstellungen, den er auf der gesellschaftlichen Ebene vollziehen will, wird auf der individuellen Ebene neuerlich von Autoren vollzogen, die den vermeintlich objektiven Wert des sogenannten "guten Lebens" dem subjektiven Wert der persönlichen Autonomie entgegenstellen. All diese Konzepte aber bleiben chiffrenhaft, solange sie nicht offenlegen, worin das konkurrierende intrinsische ,Gute" bestehen soll, und wie es in Abhebung gegen den intrinsischen Wert der ,wesensgemäßen Selbstentfaltung" begründet wird.

Mehr noch. Es stellt sich die Frage, ob der vermeintliche Konkurrenzwert, näher betrachtet, nicht selbst auf die Freiheit als den zentralen zurückführt. Die Probe darauf läßt sich wie folgt anstellen. Es muß, wären diese Begriffe tatsächlich irreduzibel, prinzipiell denkbar sein, daß das freiheitsrelativierende „Glück“, „Wohl" oder sonstige "Gute" für einen Menschen höherwertig ist, obwohl feststeht, daß er sich, wenn er ihm folgt, nicht "wesensgemäB entfaltet“. Ich will das gegenwärtig nicht völlig ausschlieBen, halte es aber für äuBerst unwahrscheinlich. Auch ein islamischer oder christlicher „Gottesstaat ${ }^{\text {* }}$ wird seinen Anspruch, bestimmte Moralvorstellungen gesamtgesellschaftlich zu erzwingen, im allgemeinen nicht nur damit begründen, daB diese göttlich geboten und deshalb absolut wertvoll sind, sondern zugleich behaupten, daB nur auf diesem Wege „wahre Freiheit" zu finden ist. Und mag dies in den Augen der Ungläubigen auch nur ein Lippenbekenntnis sein oder totale Begriffsrabulistik à la Huxley (vgl. S. 760), so zeigt selbst dies noch die

$25 \mathrm{Vgl}$ die Quellenangaben in Anm. 16.

26 H. v. Treitschke, Die Freiheit, in: ders., Ausgewählte Schriften, Bd. I, Leipzig 1907, $14 \mathrm{f}$. 
Abhängigkeit des proklamierten Konkurrenzwerts vom intrinsischen Wert der Freiheit. Ähnliches dürfte für alle spezifizierten Konzepte des Guten gelten, die man mit halbwegs plausiblem Anspruch auf Allgemeingültigkeit als Konkurrenzwerte zur Freiheit einführen kann. Durch sie ist die Unhintergehbarkeitsthese, wie es scheint, generell nicht zu erschüttern.

\section{Positive und negative Freiheit}

Weniger leicht als die explizite allerdings läßt sich die implizite Relativierung ausschließen, die dadurch entsteht, daß der Freiheitsbegriff selbst durch andere Werte besetzt wird. Könnte es deshalb sein, daß die Unhintergehbarkeit nur ein Schein ist? Ich habe hier nicht den Raum, dies eingehender zu untersuchen, möchte aber an einem einschlägigen Beispiel verdeutlichen, warum ich auch in dieser Hinsicht skeptisch bin. Ich wähle dazu die geläufige Unterscheidung zwischen ,positiver" und ", negativer Freiheit". Diese ist insbesondere durch die bekannte und vielzitierte Arbeit von Isaiah Berlin ${ }^{27}$ so etwas wie intellek tuelles Gemeingut geworden. Berlin hat sie jedoch begrifflich äußerst unscharf gefaßt und damit viel Verwirrung gestiftet. Wir müssen deshalb die wichtigen Punkte zunächst herausarbeiten.

Grob gesagt geht es um folgenden Gegensatz. Anwälte der „positiven Freiheit": meinen, $\mathrm{da} B$ dieser eigentlich entscheidende Freiheitsbegriff eine „nur negative "Freiheit wertmäßig relativiert und uberwiegt. Die Vertreter der ,negativen Freiheit" dagegen glauben, daß ihre Gegner fremde Gesichtspunkte in den Freiheitsbegriff eintragen. Wäre das letztere richtig, so wären wir damit, offenbar auf einen Wert gestoBen, der den Wert der Freiheit extern relativieren kann. Doch so allgemein, wie es hier formuliert ist, läßt sich die Frage der externen Relativierbarkeit des Wertes der Freiheit durch Konkurrenzwerte, die implizit in „positiven"Freiheitsbegriffen enthalten sind, nicht entscheiden. Denn die Unterscheidung zwischen "positiver" und ,negativer Freheit" ist mehrdeutig und präzisierungsbedürftig. Drei Deutungen vor allem sind hier hervorzuheben.

Teilweise wird der Unterschied zwischen ,positiver" und "negativer Freiheit" mit einem vermeintlichen Gegensatz zwischen „Freiheit von" und "Freiheit $z u^{*}$ in Verbindung gebracht. Ein solcher aber ist inexistent und nur die Folge einer Begriffsverwirrung. Der Rekurs auf den Gattungsbegriff der Hindernisfreiheit macht klar, daß es sich lediglich um zwei Seiten ein und derselben Sache handelt. Wer frei ist von relevanten Hindernissen, besitzt eben damit die Freiheit $z u$ den betreffenden, unverschlossenen Möglichkeiten, und umgekehrt. Diese Unterscheidung also ist insignifikant.

Ähnlich steht es mit dem vermeintlichen Gegensatz zwischen dem „Positivum“ des Tuns und dem „Negativum" des Unterlassens. Im Strafrecht, aber auch in der Moral ist man z. T. bis heute geneigt, beide Formen des Tätigseins prinzipiell voneinander zu trennen, obwohl handlungstheoretisch schärfer blickende Philosophen (wie etwa Leibniz) hier schon seit langem Klarheit geschaffen haben. Titn ist, vereinfacht gesagt, das willensabhängige Herbeifuhren eines Sachverhalts „p" (z. B. vorhandener Druck auf dem Bremspedal) als Folge einer willensabhängig hervorgebrachten, elementaren Handlung , h" ( $z$. B. Strecken des rechten Fußes). Unterlassen ist das willensabhängige Herbeiführen eines Sachverhalts

$27 \mathrm{Vgl}$. den in Anm. 24 zitierten Aufsatz. 
„-p" (fehlender Druck auf dem Bremspedal) als Folge einer willensabhängig nicht verwirklichten, elementaren Handlung, ,- $h^{*}$ (Nichtstrecken des rechten Fußes). Beide Arten von Sachverhalten können dann ihrerseits die Bedingung sein, daß ein anderer, ebenfalls positiv oder negativ zu beschreibender Sachverhalt "q" oder "-q" eintritt (z. B. Abbremsen oder Nichtabbremsen des Autos). Ein Sachverhalt dieses Typs, der nur indirekt durch menschliches Tun oder Unterlasen herbeigeführt wird, ist es zumeist, auf den sich moralische bzw. rechtliche Verbote oder Gebote wesentlich richten. ${ }^{28}$

Ein Beispiel aus dem Rechtsbereich mag das verdeutlichen. Wenn es rechtlich geboten ist, daß jeder Burger sich so verhält, daß kein anderer durch ihn zu Schaden kommt, kann das - je nach Umständen - Tätigkeiten genauso wie Unterlassungen nach sich ziehen. Man muß es z. B. unterlassen, auf andere einzuprügeln oder den Stecker aus einer HerzLungen-Maschine herauszuziehen. Ebenso aber ist man verpflichtet, einem Verletzten am Straßenrand tätige Hilfe zu leisten oder aktiv auf die Bremse zu treten, um ein vors Auto laufendes Kind nicht anzufahren. Wenn man hier einen Unterschied macht und bestimmte Tätigkeiten von solchen Verpflichtungen ausnimmt oder an eine spezielle "Garantenstellung" des Handelnden bindet, so nur deshalb, weil man bestimmte Rechtsgüter nicht für absolut, also uneingeschränkt schützenswert hält oder weil man nicht alle denkbaren Mittel von allen Normadressaten fordern möchte. Von einem prinzipiellen Unterschied zwischen „bloßem Zulassen" und, ,aktivem Herbeiführen“ kann keine Rede sein. Deshalb ist auch die Unterscheidung zwischen einem Zustand der ,negativen sozialen Freiheit", der durch Verbote sichergestellt wird, und einem Zustand der "positiven Freiheit", der auf Geboten beruht, sachlich gegenstandslos bzw. als ein Versuch zu werten, inhaltliche Differenzen darüber, welche Freiheiten schützenswert sind, scheinformal zu verschleiern.

Begrifflich signifikant ist die Unterscheidung allenfalls, wenn mit ,negativer Freiheit" das Nichtbestehen von Hindernissen gemeint ist, die den Zugang zu relevanten Teilen des Möglichkeitsspielraums prinzipiell ausschließen, und mit „positiver Freiheit" das Bestehen der Moglichkeit, diese Optionen auch konkret.d. h. aus einer bestimmten Situation heraus zu ergreifen. Das freie Individuum muß hier also nicht nur die abstrakte Chance, sondern die konkrete Fahigkeit und Gelegenheit zu den betreffenden Optionen haben. Der Unterschied ist natürlich ein gradueller. Zwischen der konkreten Freiheit eines Schlaraffenlandes, in dem jedem, der es möchte, die gebratenen Tauben von selbst in den Mund fliegen, und der abstrakten Freiheit eines Landes, in dem niemand Zugang zu solchen Genüssen hat, obwohl sie prinzipiell verfügbar wären, gibt es alle Arten von Zwischenstufen. In einer umfassenden Freiheitstheorie müßten diese natülich eingehender differenziert werden. Uns interessiert nur die Stelle, an der die Grenze zwischen ,negativer" und ,positiver Freiheit" zu ziehen ist.

Vertreter einer rein ,negativen Freiheit" im fraglichen Sinne begnügen sich mit der prinzipiellen Zugänglichkeit oder halten sich doch zumindest ans amtere Ende der Gradskala. Dazu gehören vor allem die Anhänger jener Spielart, oder besser gesagt jener Schwundstufe des Liberalismus, die sich im Kern auf die Forderung nach einer Minimierung staatlicher Interventionen beschränkt. Für sie ist der prinzipielle, hinderungsfreie Zugang zu den betreffenden Möglichkeiten genau dann gegeben, wenn diese nicht durch Gesetze oder

$28 \mathrm{Vgl}$. dazu und zum nächsten Absatz G. Seebaß. Handlungstheoretische Aspekte der Fahrlässigkeit, in: Jahrbuch für Recht und Ethik 2 (1994), $381 \mathrm{ff}$. 
sonstige Zwangsmaßnahmen verschlossen sind. Und mit diesem Konzept negativer Freiheit verbindet sich dann der Anspruch, daB positive Freiheitsbegriffe, die auf konkrete Zugänglichkeit abstellen, begriffliche Zwitter sind, die das zentrale Merkmal der Freiheit mit anderen Merkmalen mischen. Ist dieser Anspruch gerechtfertigt? Und wenn ja, könnte man dann im Gegenzug nicht behaupten, daß der Wert der in sich rein ,negativen " Freiheit extern relativiert wird durch Werte, die zwar zu Unrecht als solche der Freiheit auftreten. aber zu Recht als eigenständige Werte?

Nun, der Rekurs auf den Gattungsbegriff der Hindernisfretheit rückt die Dinge auch hier zurecht. Auch der Begriff der ,positiven Freiheit" erweist sich als reiner Freiheitsbegriff, vorausgesetzt jedenfalls, daß nicht nur die Abwesenheit von gesetzlichen Restriktionen und sonstiger öffentlicher oder privater Zwangsgewalt, sondern auch der Besitz von Gelegenheiten und Fähigkeiten eine Voraussetzung dafür sein kann, daß Menschen sich ,wesensgemäß entfalten". Dies ist der Hintergrund für die Vehemenz der Attacke, die Friedrich von Hayek, wie wir gesehen hatten (S. 761), auf das traditionelle Konzept der Handlungs- und Hindernisfreiheit führt. Hayek ist kein Vertreter eines reinen Schwundstufenliberalismus. Aber er ist ein hartgesottener Wirtschaftsliberalist, der, weil er sich gern als "Anwalt der Freiheit" verstehen möchte, nicht zugeben kann, daß auch der Mangel an Fähigkeiten, Besitz oder sonstigen Machtmitteln freiheitsbeschränkend ist. Und da er sieht, daß dies auf der Basis des Kernbegriffs der Hindernisfreiheit nicht zu haben ist, sucht er diesen Begriff verbal beseite zu drücken und den, wie er glaubt, wahren Freiheitsbegriff auf die Abwesenheit von intentionalem Zwang durch andere Menschen zu beschränken ${ }^{29}$ Hier also kann man die interessenbedingte Verbiegung des Positiv-Wortes „Freiheit" direkt miterleben.

In Wirklichkeit ist die freiheitsbeschränkende Bedeutung fehlender Fähigkeiten und Macht, okonomischer wie politischer, evident. Man muß auch kein Marxist oder sonstiger Sozialist sein, um das zu erkennen. Haben doch Autoren, die solcher Neigungen so unverdächtig und untereinander so verschieden sind wie Max Weber und Papst Pius XII., diese Bedeutung ausdrücklich anerkannt. ${ }^{30}$ Mehr noch. Es ist blanker Zynismus, einem Arbeitslosen in Leuna oder einem Jugendlichen aus den Slums von Rio zu erklären, sie seien so frei wie die Reichsten im Lande, da der Staat sie ja nicht daran hindere, durch Gründung eines Konzerns oder Übernahme eines lukrativen Aufsichtsratspostens Multimillionăr zu werden.

Ahnliches gilt für andere Machtfaktoren, allen voran das Wissen, das Aristoteles bekanntlich als eines von zwei zentralen Kriterien der Freiheit überhaupt eingeführt hat. ${ }^{31}$ Die Signifikanz dieser, ,positiven "Bedingung der Ungehindertheit kann nur deshalb leicht übersehen werden, weil sie, anders als ökonomische Macht, auf der Ebene der bloßen

$29 \mathrm{Vgl}$. Hayek. The constitution of liberty, a. a. O., 11 f, $20 \mathrm{f}, 133 \mathrm{ff}$.

$30 \mathrm{Vgl}$. M. Weber. Wirtschaft und Gesellschaft Kap. VII (= Rechtssoziologie). \$ 2 am Ende: Pius XII., Nuntius Radiophonicus, I. Sept. 1944, in: Acta Apostolica Sedis 36 (1944), 252. - Mit speziellem Bezug auf die Vertragsfreiheit hat Weber (ebd.) sogar geltend gemacht, daß, .eine formell noch so viele ,Freiheitsrechte $[\ldots]$ verbürgende $[\ldots]$ und noch so wenig Gebots- und Verbotsnormen enthaltende Rechtsordnung [... ] in ihrer faktischen Wirkung einer quantitativ und qualitativ sehr bedeutenden Steigerung nicht nur des Zwanges überhaupt, sondern auch einer Steigerung des autoritären Charakters der $Z$ wangsgewalten dienen" kỏnne.

31 Aristoteles, Nikomachische Ethik, $111,1-3 ; \mathrm{V}, 10$. 
Handlungsfreiheit noch nicht hervortritt, sondern erst auf der Ebene der Willensfreiheit. Um es an einem extremen Beispiel zu verdeutlichen: Wenn unser Lotteriesystem tatsächlich, wie es gemeint ist, auf dem Zufallsprinzip beruht, kann niemand den Ausgang der nächsten Ziehung voraussagen. Gleichwohl könnte ein Schwundstufenliberalist, der nur an der prinzipiellen Zugänglichkeit des Möglichkeitsspielraums durch willensabhängiges Handeln orientiert ist, dem Arbeitslosen in Leuna oder dem Jugendlichen in Rio entgegenhalten, sie seien selbst daran schuld, daß sie nicht Millionäre seien. Denn hätten sie letzte Woche die Zahlenkombination angekreuzt, die vorgestern gezogen wurde, wäre ihnen ein Millionengewinn sicher gewesen. Sie hätten es eben nur wollen müssen.

Diese Erklärung ist wohl noch zynischer als der Hinweis auf die theoretische Möglichkeit einer Millionärsexistenz als Aufsichtsrat oder Konzerngründer. Sie macht jedoch an einem Extremfall besonders deutlich, was auch für weniger extreme, praktisch bedeutsamere Fälle gültig ist. Wer nicht über das nötige Wissen verfügt oder nicht über die nötigen Mittel, um sich das Wissen anderer zu erkaufen, hat auch zu den Teilen des sozialen Möglichkeitsspielraums keinen Zugang. die prinzipiell allen staatlich gesichert sind. Er wird beim Abschluß von Handels- und Arbeitsverträgen von seinen gewitzteren Partnern ebenso übervorteilt werden wie vom Staat, dem er, weil er die Rechtslage nicht kennt und weder Geld noch Zeit für Prozesse hat, unnötig viele Steuern zahlt oder allzu rasch nachgibt, wenn dieser ihm mit ungerechtfertigten judiziellen und exekutiven Ansprüchen entgegentritt. Durch seine Unkenntnis oder Mittellosigkeit ist er in seiner Freiheit eingeschränkt. Dies unter Berufung auf den angeblich rein negativen Sinn von „Freiheit“ zu bestreiten oder sogar noch als ,Liberalismus“ auszugeben, ist reine Begriffsrabulistik und Demagogie. Wer diese Form der Freiheit verteidigt, versteht darunter, wie es der amerikanische Präsident Lincoln mit unübertroffener Klarheit formuliert hat, offenbar nicht länger, daß ,jeder mit sich und dem Produkt seiner eigenen Arbeit verfahren kann, wie er will“", sondern daß ,einige mit anderen Menschen und den Produkten von deren Arbeit verfahren können, wie sie wollen".,32

Diese Überlegungen zeigen auch, daß die meisten der vielumstrittenen sogenannten „sozialen Leistungsrechte" nicht auf anderen Werten beruhen, sondern sich als gewöhnliche Freiheitsrechte verstehen lassen. Die Gewährleistung eines Minimaleinkommens für alle z. B. oder subventionierte Rechtsberatung für Unbemittelte sind eines Staats, der seine Orientierung am hohen Gut der Freiheit ernst nimmt, ebenso würdig wie ein Schulsystem, das Kenntnisse zu vermitteln sucht, die das freiheitsrelevante Können der Bürger vergrößern, also z. B. Grundkenntnisse im Arbeitsrecht, Mietrecht oder Verbraucherschutzrecht.

Natürlich ist damit nicht gesagt, daß alle denkbaren Leistungsrechte gewährt oder von einem freien Staat angestrebt werden sollten. Selbstverständlich gilt es hier abzuwägen. Ob reduzierte Freiheitsspielräume auf mangelnde Leistungsbereitschaft oder früheres freies Handeln zurückgehen, ist differenzierend in Rechnung zu stellen. Und auch bei unverschuldeten oder naturgegebenen Differenzen des nutzbaren Möglichkeitsspielraums (Erdbeben, Hochwasser) kann man sinnvoll darüber streiten, wie weit deren Ausgleich eine Gemeinschaftsaufgabe ist. Begrifflich ist hier nichts vorentschieden. Wichtig ist nur, sich

32 A. Lincoln. Address at sanitary fair in Baltimore, April 18, 1864, in: A.B. Lapsley (ed.), The writings of Abraham Lincoln, vol. VII, New York/London 1906, 121; Übersetzung vom Verfasser. 
klarzumachen, daß es auch dabei in den meisten Fällen, wenn nicht in allen, nur um ein Abwägen zwischen verschiedenen Freiheiten geht, nicht zwischen dem Wert der Freiheit und anderen Werten, und daB die Notwendigkeit einer Abwägung nicht bedeuten kann, bestehende Unfreiheit nicht mehr beim Namen zu nennen.

\section{Die offene Frage der externen Relativierbarkeit}

Die Unterscheidung zwischen ,negativer" und „positiver Freiheit“" liefert uns also auch in der Form, in der sie einen signifikanten Unterschied anspricht, keinen Anhaltspunkt dafür, daß der Wert der Freiheit durch andere Werte, die implizit mit der Rede von ,Freiheit" verbunden sind, extern relativiert wird. Es wäre reizvoll, weitere Redeformen auf diese Möglichkeit hin zu prüfen. Interessant erscheinen hier insbesondere zwei: (1) die vielumstrittene, durch Hegel angeregte Formel von Engels, wonach Freiheit „Einsicht in die Notwendigkeit" sei, ${ }^{3,3}$ und (2) die schon zu Anfang erwähnte ältere These, daB wahre Freiheit in der Gebundenheit an andere Personen besteht, wobei es sich sowohl um Individuen, mit oder ohne autoritativen Führerstatus, handeln kann wie auch um Kollektive. Beide Begriffsbestimmungen sind prima facie paradox. Sie müssen deshalb den Verdacht erregen. daß fremde Gesichtspunkte in ihnen leitend sind, die, soweit sie reale Werte ansprechen, zu einer externen Relativierung der Freiheit führen.

Ob das tatsächlich so ist, steht dahin. Denn bei Hegel ebenso wie in der religiösen Tradition, wo die Gebundenheitsthese vorzüglich beheimatet ist, ${ }^{34}$ finden sich schlimme (und zu Recht scharf kritisierte ${ }^{35}$ ) Dokumente eines autoritären, kollektivistisch geprägten Untertanengeistes, der als Konkurrenzwert zu dem der Freiheit gewiß nicht in Frage kommt. Dennoch scheinen mir beide Bestimmungen. die Gebundenheitsthese wie die Formel von der Einsicht in die Notwendigkeit, etwas getroffen zu haben. Man muß sie nur aus ihrer verwirrenden (und teilweise auch begrifflich verwirten) Einkleidung lösen. Tut man dies jedoch, wird deutlich, daB wir es hier durchaus nicht mit Werten zu tun haben, die in Konkurrenz zum Wert der Freiheit stehen, sondern die ihm im Gegenteil konzeptuell untergeordnet sind.

Ich kann darauf in diesem Aufsatz nicht mehr eingehen, sondern muß den Nachweis, da $B$ es so ist, einer anderen Gelegenheit vorbehalten. Bis dahin mag der zweifelnde Leser skeptisch bleiben. Und naturlich rechne ich auch damit, daß manche Leser durch die vorstehenden Überlegungen nicht überzeugt sind, daß der Wert der Freiheit in keiner Weise,

33 F. Engels, Herrn Eugen Dühring's Umwälzung der Wissenschaft, Abschnitt 1, Kap. XI (MEW Bd. 20, Berlin 1973, 106); G. W. F. Hegel, Enzyklopädie der philosophischen Wissenschaften, $\$ 513-515$; vgl.: Grundlinien der Philosophie des Rechts, \$142-145.

34 Die Belege dafur sind zahllos. Extreme Beispiele zitiert W. James, The varieties of religious experience, New York 1902, 310-315. Weniger extreme, aber nicht weniger signifikante Außerungen finden sich z. B. bei Luther, De servo arbitrio, WA XVIII, 635; K. Barth, Die kirchliche Dogmatik, Bd. III/3, Zürich 1961, 170; D. Bonhoeffer, Widerstand und Ergebung, Stuttgart 1951, 271 f. 175 f., und H. Küng, Arbeit und Lebenssinn angesichts von Wertewandel und Orientierungskrise. in: Arbeit der Zukunft - Zukunft der Arbeit, hg. v. d. A.-Herrhausen-Gesellschaft, Frankfurt/M./Stuttgart 1994, 25.

35 Vgl. etwa die einschlägige Hegel-Kritik von E. Tugendhat, Selbstbewußtsein und Selbstbestimmung, Frankfurt/M. 1979, 345-351. 
weder explizit noch implizit, relativierbar ist. Ich wäre gespannt, plausible Konkurrenzwerte benannt zu bekommen; d. h. genauer gesagt Werte, bei denen man darauf rechnen kann, daß ihre Eigenständigkeit und Höherrangigkeit bzw. Gleichrangigkeit gegenüber dem Wert der Freiheit kritischer Prüfung standhält. Bislang zweifle ich an ihrer Existenz, lasse mich gegebenenfalls aber gern vom Gegenteil überzeugen. Nur sollte niemand sich diesen Nachweis dadurch vereinfachen, daß er anstelle des Gattungsbegriffs der Hindernisfreiheit, der auf die ,wesensgemäße Selbstentfaltung“ in einem Spielraum offener Möglichkeiten abstellt, einen im Kern verengten, sinnentleerten oder gar rabulistisch verdrehten Freiheitsbegriff zugrunde legt. Allzu leicht aufzuopfernde oder wertmäßig zu relativierende Redeformen von "Freiheit" mïssen jedenfalls den Verdacht wecken, keine Freiheitsbegriffe mehr zu sein. Denn Freiheit, wir wissen es schließlich, ist ein hohes Gut.

Prof. Dr. Gottfried Seebaß, Universität Konstanz, Philosophische Fakultä, Fachgruppe Philosophie, Postfach 5560, D-78434 Konstanz. 